\title{
LEVERAGED BENDING FOR FULL-GAP POSITIONING WITH ELECTROSTATIC ACTUATION
}

\author{
Elmer S. Hung and Stephen D. Senturia \\ Massachusetts Institute of Technology \\ Cambridge, MA 02139
}

\begin{abstract}
The pull-in instability limits the travel distance of an elastically-suspended parallel-plate electrostatic actuator to about $1 / 3$ of the gap. In this paper we report an electrostatic actuator design which can achieve complete gap travel without pull-in. The specific structure which has been fabricated and tested is designed for positioning mirrors in a tunable diffraction grating known as a polychromator; however, the actuation principle, which we call "leveraged bending," should be applicable to a wide range of devices.
\end{abstract}

\section{INTRODUCTION}

The use of electrostatic actuation for microelectromechanical systems (MEMS) is attractive because of the high energy densities and large forces available in microscale devices. In many electrostatic actuators, the positions of electrodes are controlled by a balance between an electrostatic attractive force and the action of a mechanical restoring force. A large range of motion for analog positioning can be extremely useful for a wide variety of tuning applications. Unfortunately, with electrostatic actuation, the interaction of a nonlinear electrostatic force with an elastic restoring force causes a "pullin" phenomenon which prevents electrodes from being stably positioned over a large distance.

In this paper we report the use of a "leveraged bending" approach to design an electrostatic actuator which can achieve complete gap travel without pull-in. The actuator is used to position mirrors in an analog-tuned reflective phase grating known as a polychromator. Polychromator design and implementation are described as a case study example of the leveraged bending technique.

\section{PULL-IN PROBLEM}

The pull-in problem can be illustrated by a simple $1 D$ model: Consider an ideal system (Fig. 1) consisting of two parallel conductive plates separated by gap $z$, with one plate fixed on the substrate and the other suspended by an elastic restoring force (a linear spring). If a voltage $V$ is applied across the plates, the attractive force, neglecting fringing, is given by:

$$
F_{e}=\frac{\epsilon_{0} A V^{2}}{2 z^{2}}
$$

where $A$ is the area of the movable plate and $\epsilon_{0}$ is the permitivity of free space. The electrostatic force is nonlinear, since it depends on $1 / z^{2}$, while the elastic restoring force,

$F_{m}=k\left(g_{0}-z\right)$, is linear with $z$, where $k$ is the spring constant and $g_{0}$ is the unactuated gap.

The equilibrium position of the plate may be found by equating the electrostatic and mechanical force [1]. As

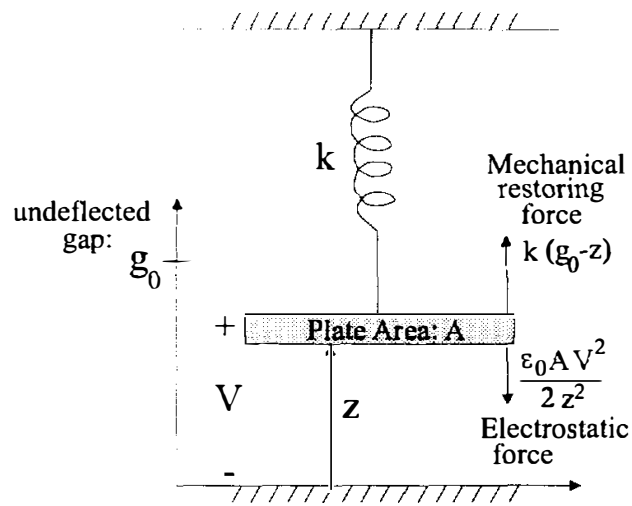

Figure 1. Ideal $1 D$ parallel plate electrostatic actuator with linear restoring force.

$V$ increases, the gap distance at first decreases gradually. However, at a critical voltage value called the pullin voltage, $V_{P I}=\frac{8 k g_{0}^{3}}{27 \epsilon_{0}}$, a bifurcation occurs and the plate suddenly collapses. The maximum stable deflection of the plate before pull-in is $1 / 3$ of the undeflected gap.

The positive feedback mechanism can be explained as follows: As the plate moves lower, more charge is drawn onto the plate to increase the electric field and keep the potential difference between the plates constant. The increase in charge and field in turn increases the electrostatic force, which pushes the plate lower again. This phenomenon is fundamental to many voltage-controlled electrostatic actuators.

There have been several previous efforts to increase the travel of electrostatic actuators without pull-in. Seeger and Crary [2] propose a stabilization technique using a matched MOS capacitor in series with an electrostatic actuator. Burns and Bright [3] use a multi-phase flexure to extend travel distance. Chu and Pister [4] report an algorithm for closed-loop voltage control of electrostatic actuators. Unlike the leveraged bending method, all three of these techniques require the fabrication of additional structures or electronics.

\section{ACTUATION PRINCIPLE}

The basic idea behind leveraged bending is to work around the pull-in instability by applying electrostatic force only on the portion of a structure, then using the rest of the structure as a "lever" to position specific parts of the structure through a large range of motion (see Fig. 2). The key is that the electrostatically-actuated portions of the structure do not have to deflect far enough to violate the pull-in limit.

Leveraged bending is best illustrated using the polychromator grating example. This application $[5,6]$ requires a linear array of long, flat, closely-packed mirrors, 


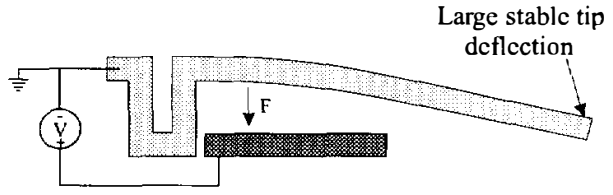

(a) Leveraged bending: cantilever

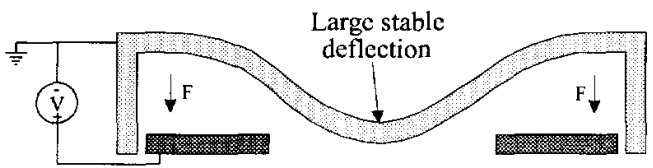

(b) Leveraged bending: Fixcd-fixed bcam (Low residual stress)

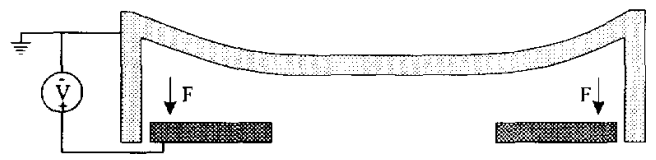

(c) Fixcd-fixcd beam (High tensilc residual stress)

Figure 2. Using leveraged bending to extend the stable travel range of an electrostatic actuator. Simple examples of leveraged bending applied to: (a) cantilevers and (b) doubly-clamped, fixed-fixed beams. (c) For fixed-fixed beams, tensile residual stress can limit the leveraged bending effect (see Eq. (4)).

each of which is vertically positioned independently. The heights of the mirrors determine the optical path length of reflected light, thus effectively controlling the optical transfer function of the grating.

Each grating element consists of a conductive doublebeam structure (Fig. 3), following the design of Bifano et al. [7], but with actuation electrodes only beneath a portion of the lower beam nearest the supports. Both beams are doubly-supported but the beams are staggered so that the flat mirror beam "rides" on top of the bottom beam, which bends under actuation (Fig. 3(b)). Voltage is applied to the actuation electrodes, and both beams are grounded. The voltage is controlled in an analog fashion to position the mirror beam vertically. A grounded landing pad between the actuation electrodes prevents welding if the beam touches down.

The bottom beam functions as a lever so that the middle of the beam moves further than the part of the beam over the actuation electrodes. It is possible to design the structure so that the middle of the beam travels the full gap before pull-in occurs.

Leveraged bending offers a number of distinct advantages for the polychromator grating design, at the cost of increased actuation voltage. First, for a given required analog travel distance, a smaller gap is needed. This is important in a surface micromachined process because thinner sacrificial layers mean shorter deposition times and less topography to complicate subsequent processing. The smaller gap distance also means less electrostatic crosstalk due to fringing fields from neighboring actuators. The dense mirror packing, large number of actuators, and fabrication process make other methods for full-gap travel difficult to implement. In addition, the design quite naturally provides for a grounded land-

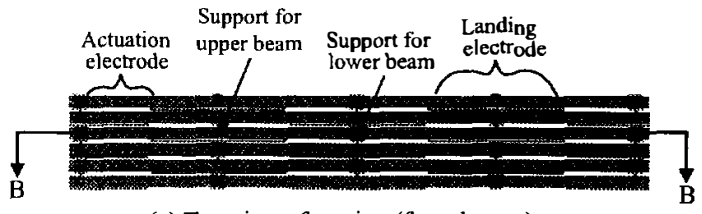

(a) Top vicw of grating (from layout)

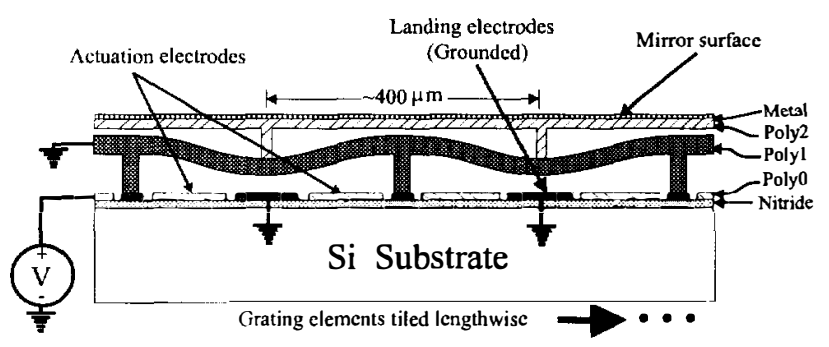

(b) Side vicw of grating (under actuation)

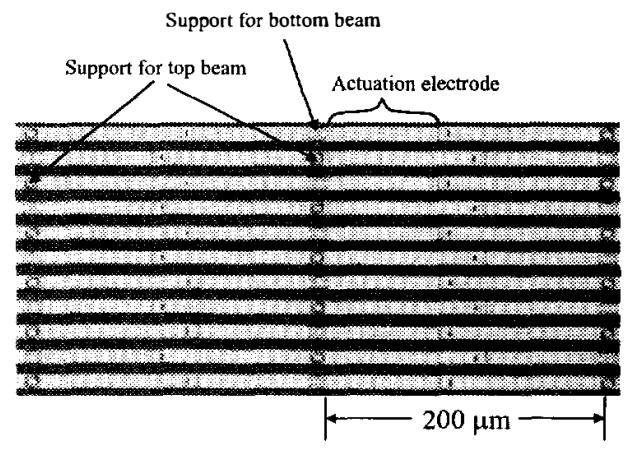

(c) Photograph of MUMPs grating

Figure 3. Polychromator diffraction grating design. (a) Top view of grating design from layout. (b) Schematic side view of grating showing a cut along the length of a single grating element (cut $B$ from (a)). The bottom beam is shown bent under actuation. The beam would be flat with no applied voltage. (c) Photograph of a MUMPs grating used for prototyping.

ing electrode which is important for eliminating stiction during operation.

Prototype gratings were fabricated using the MUMPs process from MCNC [8]. The design and analysis in this paper focus on the MUMPs polychromator grating example.

\section{ACTUATOR MODELING}

Accurate, efficient models are necessary to explore the design space of the actuator. Since the upper mirror beam does not affect actuation to first order, the grating element is modeled by considering just the lower beam. The $2 D$ equilibrium equation for an elastic beam with electrostatic force is given by:

$$
E I \frac{\partial^{4} z}{\partial x^{4}}-S(z) \frac{\partial^{2} z}{\partial x^{2}}=F_{e l e c}
$$

where $x$ is the distance along the the beam, $z(x)$ is the gap height, $E$ is the elastic modulus, $I=w h^{3} / 12$ is the moment of inertia, $w$ is the beam width, $h$ is the beam thickness, $S(z)$ is the force in the beam due to stress 
(see Eq. (3)), and $F_{\text {elec }}$ is the electrostatic force per unit length. Neglecting fringing fields we approximate:

$$
F_{\text {elec }}=-\frac{\epsilon_{0} a(x) V^{2}}{2 z^{2}}
$$

where $a(x)$ represents the shape of the actuation electrode and $V$ is the applied voltage. The $S(z)$ term contains two contributions, one from the residual stress left in the beam after processing, and one from a nonlinear "strain-stiffening" effect due to the stretching of the beam as it deforms. $S(z)$ is modeled as follows:

$$
S(z)=h w\left[\sigma_{0}(1-\nu)+E \frac{\Delta L}{L}\right]
$$

where $\sigma_{0}$ is the biaxial residual stress, $\nu$ is the Poisson ratio, $\Delta L$ is the elongation in the length of the beam due to deformation, and $L$ is the length of the beam. Note that the nonlinear strain stiffening by itself increases the travel distance of electrostatic actuators beyond 1/3 of the gap. The ends of the beam are assumed to have perfectly clamped boundary conditions. The beam equation is simulated using a finite difference code.

\section{DESIGN TRADEOFFS}

The design space for the grating actuator involves three key parameters: the length of the beam $L$, the actuation electrode length, $L_{1}$, and the residual stress, $\sigma_{0}$, in the bottom beam.

The choice of beam length, $L$, is primarily determined by fabrication constraints, since actuation voltage decreases and travel distance increases with increasing $L$. For the MUMPs process, $L=400 \mu m$ was chosen for baseline design (a relatively long beam with good yield).

The choice of actuation electrode length, $L_{1}$, represents a trade-off of travel distance for actuation voltage. Shorter actuation electrodes increase the travel distance but also increase actuation voltage.

For example, assuming $L=400 \mu m$, suppose we would like to pick the length of the actuation electrode, $L_{1}$. Fig. 4 shows a simulation illustrating the tradeoff between actuation voltage and travel distance for varying actuation electrode length, $L_{1}$. Curves extending from the upper left to lower right show that pull-in voltage (left axis) is reduced for larger $L_{1}$. Curves extending from the lower left to upper right show that the beam deflects further at pull-in (right axis) for smaller $L_{1}$. The two different curves represent traces for different values of residual stress (for MUMPs, typical residual stress is about -10 $\mathrm{MPa})$.

Based on Fig. 4, $L_{1}=80 \mu m$ was chosen for the baseline actuation electrode length in order to get full-gap travel while minimizing actuation voltage. Full-gap actuation requires $108 \mathrm{~V}$, about 6.1 times higher than the pull-in voltage of a similar beam with a full actuation electrode. Note, however, that the ratio between full-gap and full-electrode actuation voltage varies significantly depending on the specific structure involved.

Residual stress in the beam is also important for grating design, particularly if the stress is tensile. Although this is not an issue with the MUMPs process, it is worth noting that a tension-dominated beam acts like a rubber band. When force is applied at the ends of the beam, the

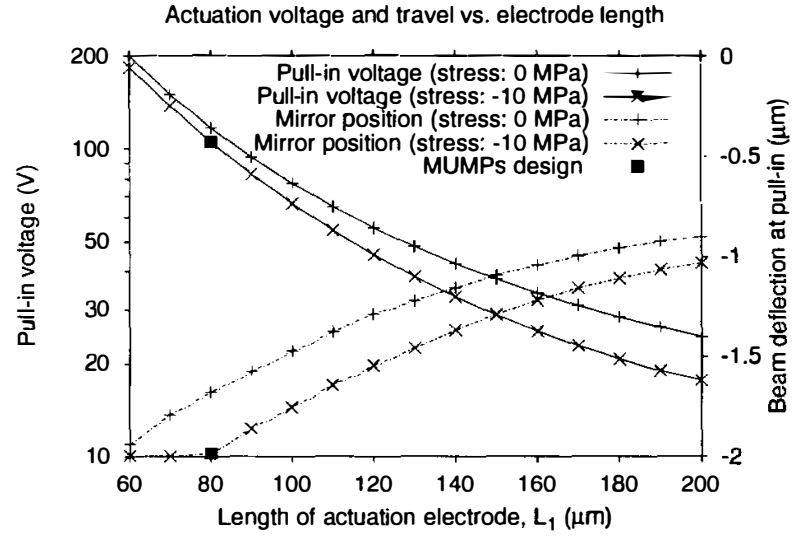

Figure 4. Finite difference simulation of the tradeoff between actuation voltage and travel distance vs. $L_{1}$ (for $L=400 \mu \mathrm{m}$, beam thickness and gap $=2.0 \mu \mathrm{m}$ ). Curves extending from the upper left to lower right show that pull-in voltage (left axis) is reduced for larger $L_{1}$. Curves extending from the lower left to upper right show that the beam deflects further at pull-in (right axis) for smaller $L_{1}$.

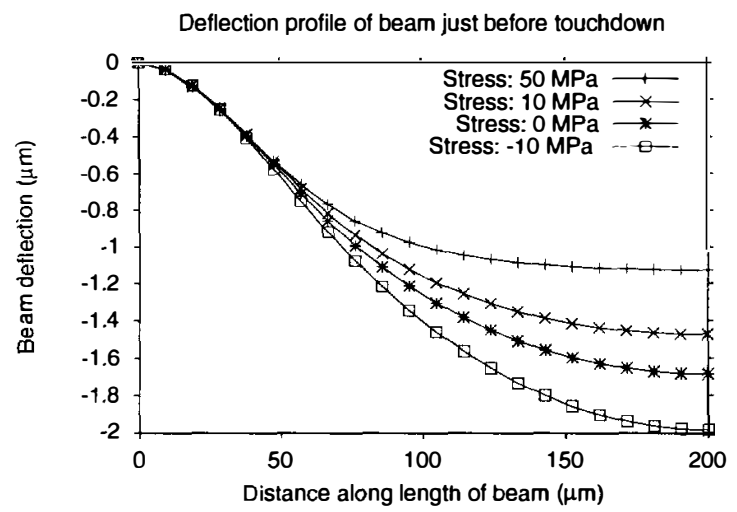

Figure 5. Finite difference simulation of the deflection profile for an $L=400 \mu \mathrm{m}$ long beam with an $L_{1}=80 \mu \mathrm{m}$ actuation electrode at the maximal deflection position before pull-in. For more tensile (positive stress) beams, travel is reduced because the middle of the beam stays flat when force is applied to the ends of the beam. For stress $=-10 \mathrm{MPa}$ (typical for MUMPs), full gap travel without pull-in is predicted.

middle of the beam remains flat, reducing the lever effect that increases travel (Fig. 2(c)). A rule of thumb for determining whether the actuator is tension-dominated can be obtained by comparing the analytical solutions [9] for Eq. (1) in the bending and tension-dominated cases, neglecting strain stiffening and assuming uniform load on the beam over the actuation electrodes. This analysis indicates that the residual stress, $\sigma_{0}$, for the fixed-fixed beam grating design must be roughly:

$$
\sigma_{0}<\frac{E h^{2}}{2(1-\nu) L_{1}\left(L-\overline{L_{1}}\right)}
$$

for leveraged bending to be useful in the fixed-fixed beam design. Thus thicker, shorter beams and shorter actuation electrodes tend to increase the tensile residual stress that can be tolerated. For the beam dimensions of the 


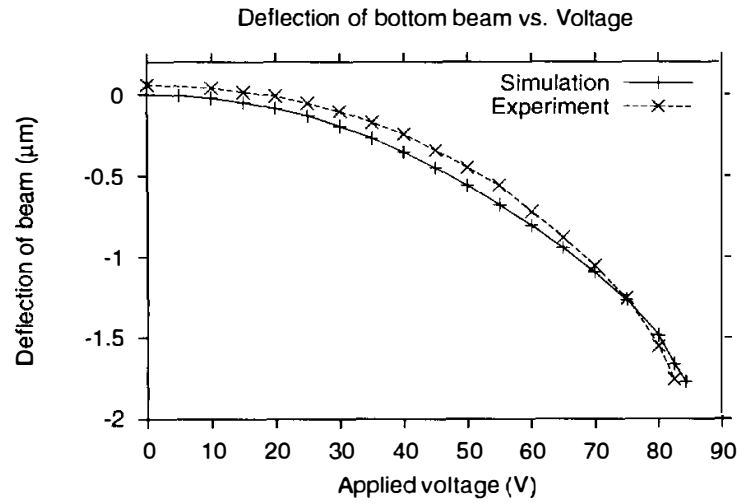

Figure 6. Graph of bottom beam deflection vs. voltage for MUMPs grating elements without the top beam $(L=400 \mu \mathrm{m}$ and $\left.L_{1}=80 \mu \mathrm{m}\right)$. More than $1.75 \mu \mathrm{m}$ of stable travel was measured out of a $2 \mu \mathrm{m}$ gap. Measurement compares well with finite difference simulation. Small discrepancies may be due to unmodeled compliant supports in the real structure. Position and film thickness measurements made using a WYKO interferometric profilometer.

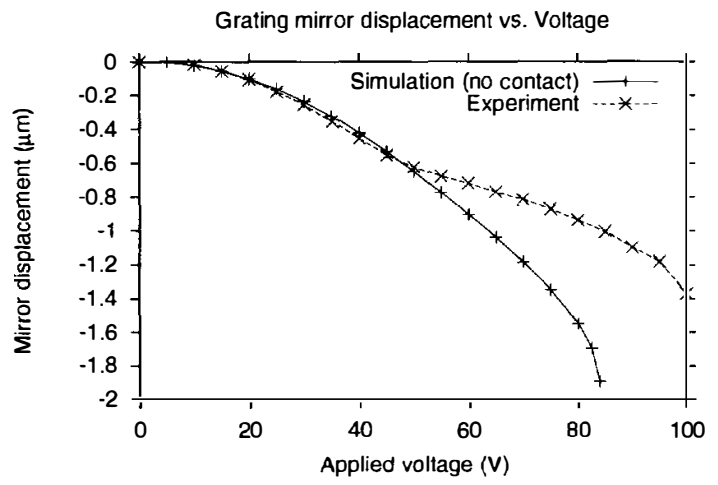

Figure 7. Graph of measured mirror position vs. voltage for MUMPs grating element. After the mirror travels $0.67 \mu \mathrm{m}$, the top beam hits the post of the bottom beam, causing the structure to become stiffer. The simulation shows what would happen without this effect.

MUMPs gratings, Eq. 4 yields $\sigma_{0}<13 \mathrm{MPa}$. This is confirmed by Fig. 5 which shows the profile of the beam at pull-in for varying stress values, illustrating how maximum travel distance decreases with tensile residual stress for the fixed-fixed beam grating design.

\section{EXPERIMENTAL RESULTS}

Position and film thickness measurements were made using a WYKO interferometric profilometer. Measurements of MUMPs structures with the bottom grating beam only (no mirror beam) demonstrate that at least $1.75 \mu \mathrm{m}$ of travel is possible out of the $2 \mu \mathrm{m}$ gap (Fig. 6). Material property values were extracted using the thickness measurements and pull-in voltage measurements of test structures as described in [1]. The extracted material properties and thicknesses were used in the simulation plotted against the experimental data in Fig. 6.

With the MUMPs process, the nominal bottom gap is $2 \mu \mathrm{m}$, while the nominal upper gap is only $0.75 \mu \mathrm{m}$.
Thus, in the full grating element, after the bottom beam deflects beyond about $0.75 \mu \mathrm{m}$, the top beam contacts the post of the bottom beam, making the structure stiffer (Fig. 7). Although not intentional, the effect is similar to the multi-phase flexures used by Burns, et al. [3]. The simulation matches the measured deflection before contact well, showing that the bottom beam model is an accurate predictor of grating element behavior. Gratings with a larger upper gap are currently being fabricated in a custom process at the Honeywell Technology Center.

\section{CONCLUSIONS}

This paper investigates the leveraged bending technique for extending the stable travel range of electrostatic actuators. As a case study example, the leveraged bending technique is used to design and implement the polychromator, a reflective phase grating with many mirrors, vertically positioned in an analog fashion.

The leveraged-bending approach offers a number of key advantages for the polychromator grating design, including ease of fabrication, less electrostatic crosstalk, and anti-stiction landing electrodes. The utility of the technique and the models on which it is based are verified with experimental measurements of deflection vs. voltage characteristics using prototype gratings.

\section{ACKNOWLEDGMENTS}

Support for this research was provided by the Defense Advanced Research Project Agency under contract N66001-97-C-8620. The authors wish to thank Bill Herb, Dave Burns, Aravind Padmanabhan, Ben Hocker, David Arch, Mike Butler, Mike Sinclair, Tony Ricco, and Erik Deutsch for useful discussions.

\section{REFERENCES}

[1] P. M. Osterberg and S. D. Senturia, "M-TEST: A test chip for MEMS material property measurement using electrostatically actuated test structures," J. Microelectromech. Syst., 6 2, 1997, pp. 107-118.

[2] J. I. Seeger and S. B. Crary, "Stabilization of electrostatically actuated mechanical devices," Transducers '97, pp 1133-1136.

[3] D. M. Burns and V. M. Bright, "Nonlinear flexures for stable deflection of an electrostatically actuated micromirror," Proc. SPIE: Microelectronics Structures and MEMS for Optical Processing III, Vol. 3226, Sept. 1997, pp. 125135.

[4] P. B. Chu and K. S. J. Pister, "Analysis of closed-loop control of parallel plate electrostatic microgrippers," Proc. IEEE Int. Conf. Robotics and Automation, May 1994, pp. 820-825.

[5] M. B. Sinclair, M. A. Butler, A. J. Ricco, and S. D. Senturia, "Synthetic spectra: a tool for correlation spectroscopy," Applied Optics, 36 15, 1997, pp 3342-3348.

[6] M. B. Sinclair, M. A. Butler, S. H. Kravitz, W. J. Zubrzycki, and A. J. Ricco, "Synthetic infrared spectra," Optics Letters, 22 13, 1997, pp 1036-1038.

[7] T. G. Bifano, et al., "Continuous-membrane surfacemicromachined silicon deformable mirror," Optical Engineering, 36 5, May 1997, pp 1354-1360.

[8] D. A. Koester, R. Mahadevan, and K. W. Markus MUMPS Introduction and Design Rules, MCNC MEMS Technology Applications Center, 1994.

[9] W. C. Young, Roark's Formulas for Stress and Strain, McGraw Hill, NY, 1989. 